\title{
Percepción del paisaje, agua y ecosistemas en la cuenca del río Dagua, Valle del Cauca, Colombia
} PERCEPTION OF LANDSCAPE, WATER AND ECOSYSTEMS IN THE WATERSHED OF THE DAGUA RIVER, VALLE DEL CAUCA, COLOMBIA

\author{
PERCEPÇÃO DA PAISAGEM, ÁGUA E ECOSSISTEMAS NA BACIA DO RIO \\ DAGUA, VALLE DEL CAUCA, COLÔMBIA
}

\author{
Marco Antonio Aguirre \\ Laura Isabel López Ibarra² \\ Francy Viviana Bolaños Trochez ${ }^{3}$ \\ Diego Fernando González Guevara ${ }^{4}$ \\ Oscar Buitrago Bermúdez ${ }^{5}$
}

Para citar este artículo: Aguirre, A. López Ibarra, L. I., Bolaños Trochez, F. V., González Guevara, D. F. y Buitrago Bermúdez, O. (2017). Percepción del paisaje, agua y ecosistemas en la cuenca del río Dagua, Valle del Cauca, Colombia. Perspectiva Geográfica, 22(1), 109-126. doi: 10.19053/01233769.5402
Recepción:

21 de octubre de 2016

Evaluación:

5 de febrero de 2017

Aprobación:

21 de marzo de 2017

\section{Resumen}

El paisaje se sitúa en el plano de contacto entre los hechos naturales y humanos, además, es un producto ecológico, psicológico y social. Su estudio permite incorporar la visión holística, dialéctica y sistémica, propia de la geografía, y se convierte en una vía para la formulación de instrumentos y políticas ambientales en la gestión y la planificación de cuencas hidrográficas. En esta perspectiva, se presenta la metodología para identificar unidades de paisajes antropogénicos, así como la percepción que los agentes sociales tienen frente a estos, el agua y los ecosistemas en la cuenca hidro-

1 Geógrafo, asistente de investigación del Grupo Territorios, Departamento de Geografía, Universidad del Valle, Colombia. geografia.777@gmail.com

2 Geógrafa, Departamento de Geografía, Universidad del Valle, Colombia. lauraisalopez12@ hotmail.com

3 Geógrafa, asistente de investigación del Grupo Territorios, Departamento de Geografía, Universidad del Valle, Colombia. Ilinfko@hotmail.com

4 Geógrafo, Departamento de Geografía, Universidad del Valle, Colombia. diego2114@hotmail.com

5 Profesor Titular Departamento de Geografía, Universidad del Valle, Colombia. Líder Grupo Territorios. Director Maestría en Desarrollo Sustentable, Universidad del Valle, Colombia. oscar. buitrago@correounivalle.edu.co 
Marco Antonio Aguirre, Laura Isabel López Ibarra, Francy Viviana Bolaños Trochez,

Diego Fernando González Guevara, Oscar Buitrago Bermúdez

gráfica del río Dagua, localizada al occidente del departamento del Valle del Cauca, Colombia. Para lo anterior, se emplearon métodos de investigación cualitativos y cuantitativos. Los resultados evidencian las condiciones de los paisajes, el agua y los ecosistemas, lo cual se convierte en un aporte para futuros procesos de planificación en la cuenca.

Palabras clave: agua, cuenca hidrográfica, ecosistemas, paisajes antroponaturales, percepción, río Dagua.

\section{Abstract}

Landscape is situated in the plane of contact between the natural and human habitats, in addition, it is an ecological, psychological and social product. Studying them allows to incorporate the holistic, dialectical and systemic vision, which is a characteristic of geography, and becomes a way for the formulation of environmental instruments and policies in the management and planning of watersheds. In this perspective, the methodology to identify units of anthropogenic landscapes is presented, as well as the perception that the social agents have against them, considering water and ecosystems in the watershed of the Dagua river, located to the west of the department of Valle del Cauca, Colombia. For this, qualitative and quantitative research methods were used. The results show the conditions of landscapes, water and ecosystems, which becomes a contribution for future planning processes in the watershed.

Keywords: water, watershed, ecosystems, anthropogenic landscapes, perception, Dagua river.

\section{Resumo}

A paisagem se situa em um plano de contato entre os eventos naturais e humanos, também e um produto ecológico, psicológico e social. Seu estudo permite incorporar a visão holística, dialética e sistêmica, própria da geografia e se converte em uma via para a formulação de instrumentos e políticas ambientais na gestão e planificação de bacias hidrográficas. Nesta perspectiva, se apresenta a metodologia para identificar unidades de paisagem antropogênicas, assim como a percepção que os agentes sociais tem frente a estes, a água e os ecossistemas na bacia hidrográfica do rio Dagua, localizada a oeste do Estado de Valle del Cauca, Colômbia. Para o interior, se utilizaram métodos de investigação qualitativos e quantitativos. Os resultados evidenciam as condições das paisagens, água e ecossistemas, o qual se converte em um aporte para os futuros processos de planificação na bacia.

Palavra-chave: água, bacia hidrográfica, ecossistemas, paisagens antropogênicas, percepção e rio Dagua. 


\section{Introducción}

La cuenca hidrográfica del río Dagua es uno de los más importantes ecosistemas productores de agua para el departamento del Valle del Cauca. Localizada sobre la vertiente del Pacífico, "es una cuenca estratégica para el país, al pasar por ella la carretera a Buenaventura, principal puerto colombiano sobre el Pacífico. Esta presenta constantemente deslizamientos que cobran vidas e interfieren con el tráfico al puerto de Buenaventura, además de aportar una significativa carga de sedimentos a la bahía de esta ciudad" (Gobernación del Departamento del Valle del Cauca et al., 2014, p. 2). Por otro lado, las actividades agropecuarias, forestales y mineras ocasionan problemas directos sobre el suelo y el agua, que sumados a la gran pluviosidad pueden afectar la población y sus actividades económico-productivas. La cuenca hidrográfica se divide en dos partes, alta y baja (Gobernación del Departamento del Valle del Cauca et al., 2014), con un área aproximada de 142.200,18 ha (Figura 1).

Los municipios con presencia en la cuenca corresponden a Dagua, con 58.439,49 ha; Buenaventura, con 33.371,97 ha; La Cumbre, con 22.199,02 ha; Restre- po, con 18.975,87 ha; Vijes, con 6.585,26 ha; Yotoco, con 2.327,71 ha, y Calima Darién, con 300,86 ha. En materia hídrica, la cuenca, en su parte alta, presenta niveles de precipitación que oscilan entre los $900 \mathrm{y}$ $1.600 \mathrm{~mm} / \mathrm{año}$, y en su parte baja, rangos de 6.000 a $8.000 \mathrm{~mm} / \mathrm{año}$. La curva hipsométrica evidencia gran acumulación del vital líquido entre los $900 \mathrm{y}$ 1.400 m. s. n. m. (ver Figura 2), cualidad morfogénica que corresponde a una cuenca triangular de origen estructural, definida como un "sistema hidrológico de respuesta rápida y de características torrenciales" (Universidad del Valle, 2016b, p. 7) que propicia un alto potencial de erosión. Esta situación contrasta con altos niveles de sedimentación y pérdida del suelo debido a sus pendientes pronunciadas, superiores al 26 $\%$ (Universidad del Valle, 2016b), y al desarrollo de actividades agropecuarias en ladera, sin control de la erosión. Después de los 900 metros, se destaca la mayor proporción de afluentes hídricos que drenan sobre el cauce principal debido a las condiciones de precipitación. En esta parte de la cuenca se localizan actividades de tipo extractivo para materiales de construcción, debido a la ampliación de la doble calzada Loboguerrero-Buenaventura.

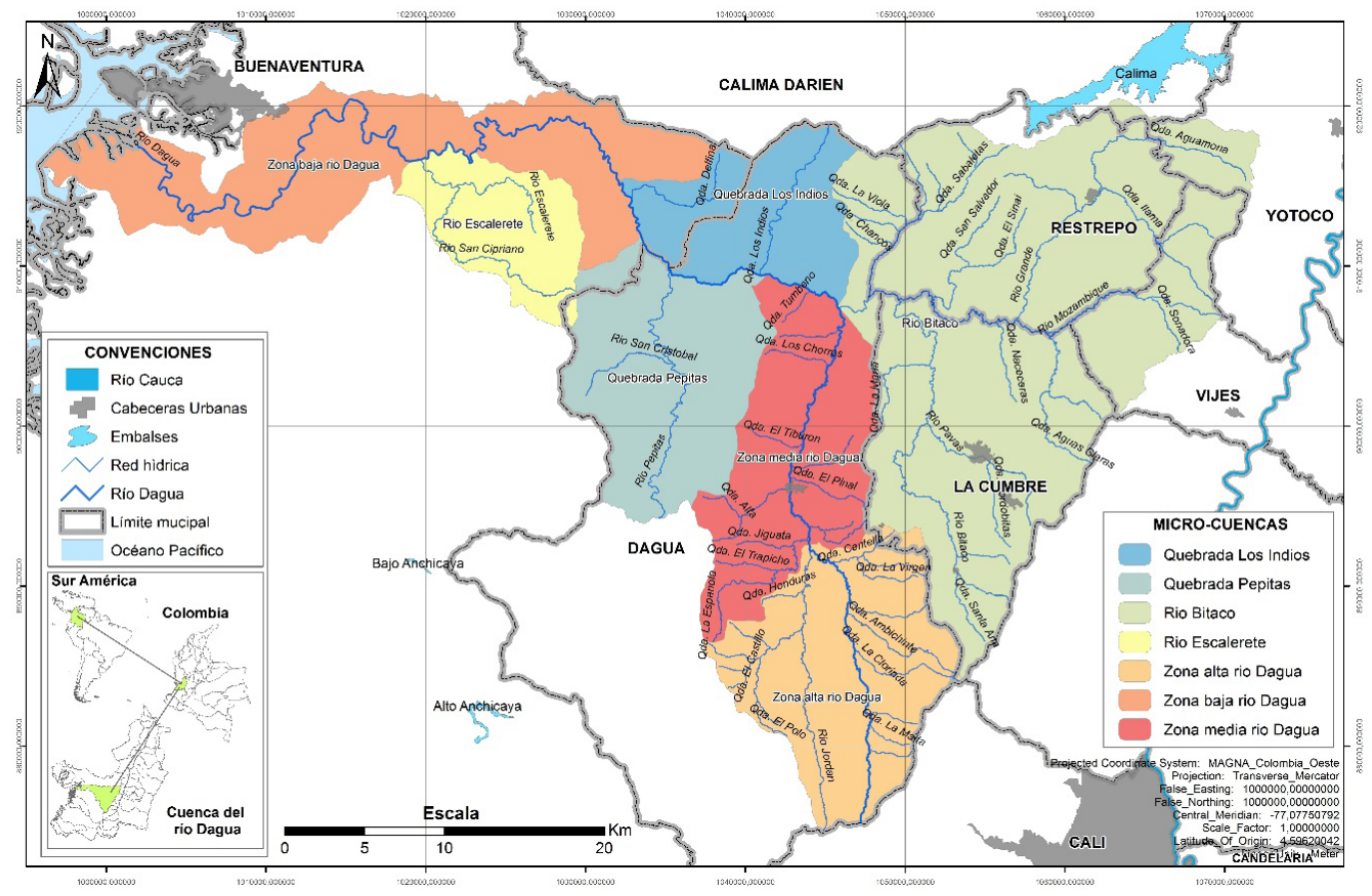

Figura 1. Localización de la cuenca hidrográfica del río Dagua.

Fuente: Elaboración propia. 
Marco Antonio Aguirre, Laura Isabel López Ibarra, Francy Viviana Bolaños Trochez,

Diego Fernando González Guevara, Oscar Buitrago Bermúdez

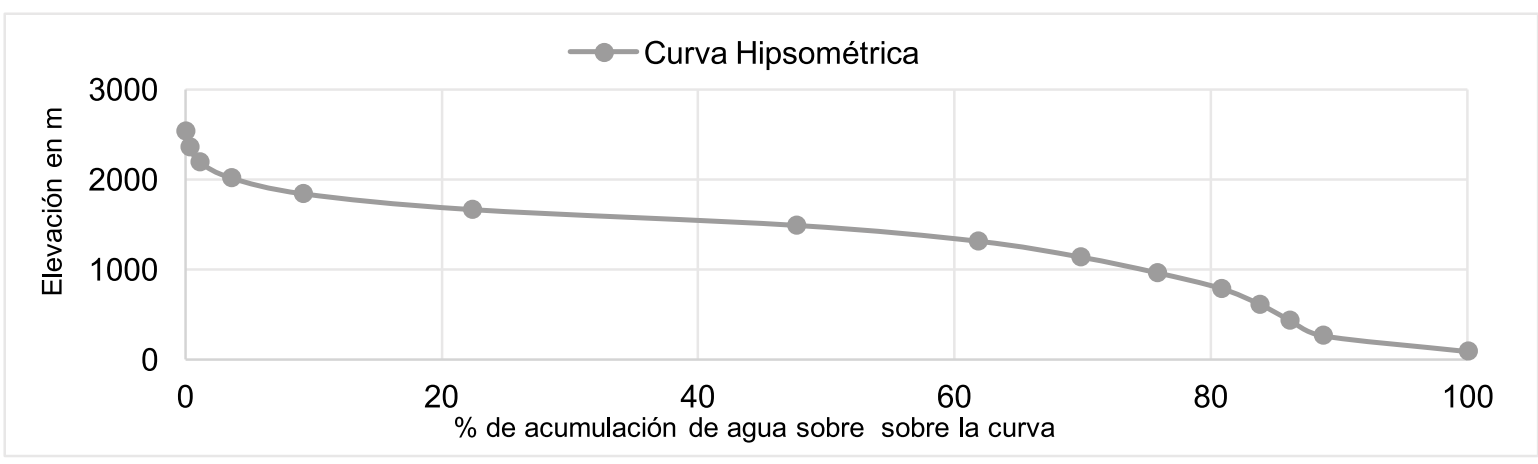

Figura 2. Curva hipsométrica de la cuenca hidrográfica del río Dagua.

Fuente: Elaboración propia.

La cuenca presenta gran diversidad de agentes sociales, entre los cuales se destacan comunidades afrodescendientes, grupos indígenas y campesinos. Según la Universidad del Valle (2016a), su dimensión espacial permite distinguir la presencia de agentes sociales de la siguiente manera: en la parte baja se ubican dos grupos de suma importancia histórica y cultural: las comunidades afrodescendientes, organizadas a través de consejos comunitarios, y los grupos indígenas, bajo la figura de resguardos, cada uno con perspectivas diferentes frente a la concepción del paisaje, el agua y los ecosistemas.

En la cuenca alta se localizan comunidades campesinas con sistemas tradicionales de producción agropecuaria, así como pequeños y medianos productores que realizan prácticas productivas semitecnificadas. Estos últimos desempeñan un rol importante en la transformación del paisaje y, a su vez, presionan el acceso al agua y los ecosistemas y generan procesos de erosión y contaminación de las fuentes hídricas (Universidad del Valle, 2016a). Del mismo modo, la parte alta concentra la mayor proporción de centros poblados municipales, con excepción de Buenaventura, Calima Darién, Yotoco y Vijes, razón por la cual aporta la mayor cantidad de aguas domésticas y residuos sólidos provenientes de actividades residenciales y productivas, las cuales se intensifican, sin control ni vigilancia de las autoridades ambientales competentes, ejerciendo presión en las zonas boscosas que aún prevalecen en la cuenca (Universidad del Valle, 2016a). Así mismo, se presentan áreas subxerofíticas con bajas precipitaciones, fuertes pendientes, suelos ácidos y redes hídricas de bajo caudal.

Por otro lado, "la parte baja de la cuenca refleja condiciones climáticas más constantes que la parte alta. Aquí se da una alta pluviosidad de aproximadamente $7.000 \mathrm{~mm} /$ año trayendo como consecuencia constantes problemas de desbordamientos, avalanchas torrenciales, pérdidas humanas, $\mathrm{y}$ arrastre de material en quebradas aledañas" (Gobernación del Departamento del Valle del Cauca et al., 2014, p. 2). La minería aurífera y de extracción de materiales de construcción son las actividades económicas con mayores impactos socioambientales en la parte baja de la cuenca; con respecto a la primera, en el 2009 se presentó un incremento en el corregimiento de Zaragoza, sin ningún tipo de control oficial (municipio de Buenaventura), causando daños irreversibles en los ecosistemas y fuentes de agua. En relación a la segunda, esta se desarrolla directamente sobre el cauce principal del río Dagua para la ampliación de la doble calzada Loboguerrero-Buenaventura (Gobernación del Departamento del Valle del Cauca et al., 2014), incrementando los problemas de sedimentación de la cuenca y afectando directamente la bahía de Buenaventura, localizada sobre el océano Pacífico.

En este contexto, a partir del campo de la geografía, resulta de interés describir, desde la visión de distintos agentes sociales, cómo las acciones humanas han incidido sobre el paisaje, afectando elementos esenciales para la vida como el agua y 
los ecosistemas en la cuenca hidrográfica del río Dagua, un asunto que debe ser considerado en los procesos de ordenación futuros de las cuencas hidrográficas en Colombia, debido a la aproximación que se realiza a las realidades locales de las distintas comunidades.

\section{Referentes teóricos}

La base teórica de la investigación se apoya en la noción de paisaje, fundamentada desde la teoría del geosistema y la geoecología del paisaje (Mateo, Da Silva y Cavalcanti, 2007). Estos enfoques permiten realizar una aproximación teórica al análisis sistémico de la categoría de paisaje, facilitando la identificación de sus principales elementos, que corresponden a unidades físico-geográficas, unidades geoecológicas y nociones sociales sobre el paisaje. Esta última integra la noción de percepción, la cual aporta bases teóricas para la comprensión de las relaciones socioespaciales y los impactos antropogénicos que modifican el paisaje e infieren en la estabilidad o degradación de los ecosistemas y los recursos naturales, tales como el suelo, el bosque y el agua. El planteamiento epistemológico se efectúa desde la perspectiva fisiográfica y morfológica de la geografía soviética, la cual plantea que "los sistemas espacio-temporales que se manifiestan en la superficie terrestre se producen a partir del intercambio de flujos y energía producto de la interacción entre la naturaleza y la sociedad" (Mateo, 2014, p. 8).

Según lo expuesto por Mateo (2008), el mundo en que vivimos es sistémico y se caracteriza por la existencia de formaciones interrelacionadas, donde los diferentes elementos forman un todo único e integral, que se distingue de su medio y se relaciona con él; en este contexto, el paisaje, como parte del sistema, comprende una categoría geográfica en la cual se desarrollan relaciones espaciales de interdependencia entre los distintos elementos integradores del paisaje que, a su vez, otorgan características propias a cada tipo de paisaje. Lo anterior complementa la perspectiva soviética, la cual se basaba en la idea de la concordancia absoluta entre todos los componentes del paisaje (base litológica, suelos, agua, masas de aire, vegetación y fauna, entre otros) que le dan sentido por su interacción e intercambios de materia y energía (Mateo, Da Silva y Leal, 2012).

En ese sentido, la geoecología de los paisajes propuesta en 1970 tiene como objetivo el estudio de las relaciones existentes en el paisaje a partir del análisis sistémico que vincula tanto elementos antrópicos como naturales, así como aspectos socioeconómicos y culturales (Mateo, Da Silva y Leal, 2012). En esencia, esta disciplina de carácter ambiental busca el diagnóstico integrado del paisaje a través de unidades geoecológicas o geoambientales con carácter individual o tipológico; cada una de estas unidades presenta cierta interrelación entre sus componentes y, por lo tanto, depende en gran medida de los procesos antropogénicos presentes en cada unidad (Mateo, Da Silva y Leal, 2012).

Según Mateo, Da Silva y Cavalcanti (2007), el paisaje es natural debido a los elementos físico-naturales que lo constituyen, es antropo-natural por la modificación que generan las actividades humanas en los espacios naturales, y es cultural porque se considera como el punto en el cual se establecen los grupos sociales en el tiempo, es decir, que en este se expresan sus actividades (económicas, reproductivas, culturales y sociales) a través del valor y la percepción que se tenga del entorno, así como a través de los usos y transformaciones que realizan en él las distintas sociedades. En este orden de ideas, analizar la percepción que un grupo de individuos tiene de su entorno contribuye no solo a establecer cómo se relacionan con este, cómo lo viven y transforman, de acuerdo a criterios preestablecidos social y culturalmente, sino también a dar cuenta de la calidad ambiental del mismo, lo cual servirá, en el corto y mediano plazo, para comprender y gestionar los recursos naturales sobre el territorio (Universidad del Valle, 2016a).

Por otro lado, existen múltiples definiciones del paisaje acuñadas en geografía y orientadas a la comprensión de las transformaciones socioespaciales y el ordenamiento del territorio, entre las que se destacan los planteamientos de Bolós y Gómez (2009), quienes afirman que "el conocimiento 
Marco Antonio Aguirre, Laura Isabel López Ibarra, Francy Viviana Bolaños Trochez,

Diego Fernando González Guevara, Oscar Buitrago Bermúdez

del estado actual del paisaje, su historial y tendencia evolutiva nos dan el punto de partida para conocer la idoneidad de los usos pasados y presentes en el proceso de producción del territorio" (p. 178). En ese sentido, Milton Santos (2000) destaca que el paisaje, como categoría de análisis dentro de la ciencia geográfica, se convierte en una herramienta importante para el entendimiento y la reflexión espacial, ya que logra contar, a través de la historia, la forma en la cual ha evolucionado y se ha transformado el espacio.

En cuanto a la percepción, es la primera forma en la cual los individuos establecen relaciones con el medio que los rodea, es la interpretación realizada de todos los componentes del paisaje, mediada por valores sociales y culturales propios del observador (Universidad del Valle, 2016a). Además, es un proceso de selección de información, de reconocimiento y de interpretación de mensajes recibidos por los órganos sensoriales, es distinta para cada individuo, depende de los rasgos culturales y propios de la personalidad y está condicionada por la capacidad de percepción de cada individuo dentro de la sociedad. Dichos factores definen la imagen que un individuo o una comunidad adquiere de su territorio y, así mismo, brinda elementos para comprender cómo las comunidades gestionan los recursos naturales. La percepción, según Arias (2003), es el eje de todas las relaciones que una sociedad/individuo establece con el entorno. Toda la información recibida por los sentidos sirve únicamente como referencia primaria para cristalizar sobre ella un complejo proceso perceptivo. Con respecto a lo anterior, se parte de la percepción del paisaje para interpretar la forma en que las comunidades localizadas en la cuenca hidrográfica del río Dagua conciben el paisaje, el agua y los ecosistemas como elementos que pueden contribuir en los procesos de planificación ambiental y territorial.

\section{Metodología}

La metodología integra técnicas cuantitativas, a partir del empleo de sistemas de información geográficos (SIG), e información de tipo cualitativo, ya que se incorporan talleres de cartografía social y entrevistas semiestructuradas con agentes sociales claves en la gestión del recurso hídrico en la cuenca. Esta se divide en dos fases (Figura 3): en la primera se llevó a cabo el proceso de revisión de literatura para definir elementos teóricos y conceptuales previos a la clasificación de unidades de paisajes antroponaturales.

Para establecer la tipología de paisajes, se contó con información secundaria del año 2004, suministrada por la Corporación Autónoma Regional del Valle del Cauca (CVC), asociada a litología, provincias de humedad, clima, curvas de nivel e inclinación de la pendiente a escala 1:25.000 para la cuenca hidrográfica del río Dagua. El geoprocesamiento de la información se realizó con el software ArcGis 9.3 disponible en el Departamento de Geografía de la Universidad del Valle. Una vez integrados los primeros elementos, se obtiene el mapa de unidades físico-geográficas para la cuenca (Universidad del Valle, 2016a).

El resultado de las unidades físico-geográficas se cruzó con información asociada a las coberturas de la cuenca para obtener el mapa de unidades geoecológicas, a las cuales se les asignó el grado de transformación antrópica (GTA) con el ánimo de calcular el índice de antropización de la cobertura vegetal en la cuenca (IACV), que mide el grado de intervención humana o la afectación en las coberturas del suelo presentes en cada unidad superior (Universidad del Valle, 2016a).

El GTA se calculó a partir del nivel de intervención antrópica por cobertura presente en la cuenca, con valores que van de 0 a 1 . Los valores por debajo de 0,2 reflejan un menor grado de transformación de las coberturas, los valores entre 0,3 y 0,6 representan transformaciones moderadas, y los valores superiores a 0,7 equivalen a altos grados de transformación en el paisaje (por ejemplo, áreas construidas, minería tecnificada, agricultura semitecnificada, monocultivos, etc.). Para definir el GTA se partió de los planteamientos teóricos de Shishenko (1988) y Priego, Bocco, Mendoza y Garrido (2008), quienes adaptaron el GTA para la cuenca de Lerma-Chapala en México; para esta investigación se contó con la valoración de expertos académicos y conocedores de la cuenca hidrográfica que, a partir de matrices, calificaron el GTA en cada cobertura. El IACV permitió definir rangos para las categorías de paisajes antropogénicos: naturales, antroponaturales y culturales, y se calculó a partir de la siguiente fórmula: 
n

\section{$\sum_{i=1} r_{i}{ }^{*} A_{i j}$}

$\mathrm{IACV}=$

\section{ATj}

Donde:

ri = grado de transformación antropogénica (GTA) de los paisajes del tipo i de cobertura.

Aij = área dedicada al tipo de utilización i en el geocomplejo j (área de la cobertura evaluada).

$\mathrm{ATj}$ = área total del paisaje $\mathrm{j}$ o la unidad de estudio (Espinoza, 2013, p. 29).
Así, se obtuvo un mapa de paisajes antropogénicos para la cuenca, que fue validado a partir del trabajo en campo previo y empleado como insumo en los talleres de cartografía social con los agentes sociales. En la fase dos se realizó la selección de agentes sociales (Lobato, 2014) a partir de un muestreo teórico o intencional (Martínez, 2012). Los agentes se escogieron a partir de tres tipologías: usuarios del agua, institucionales y sociedad civil (Massochini, 2012). Con el marco teórico y los agentes sociales definidos, se establecieron categorías de análisis para las entrevistas, las cuales corresponden a la percepción y el cambio del paisaje y las condiciones ambientales del agua y los ecosistemas, asociados principalmente a su calidad visual a raíz de los efectos generados por las actividades económicas y productivas.

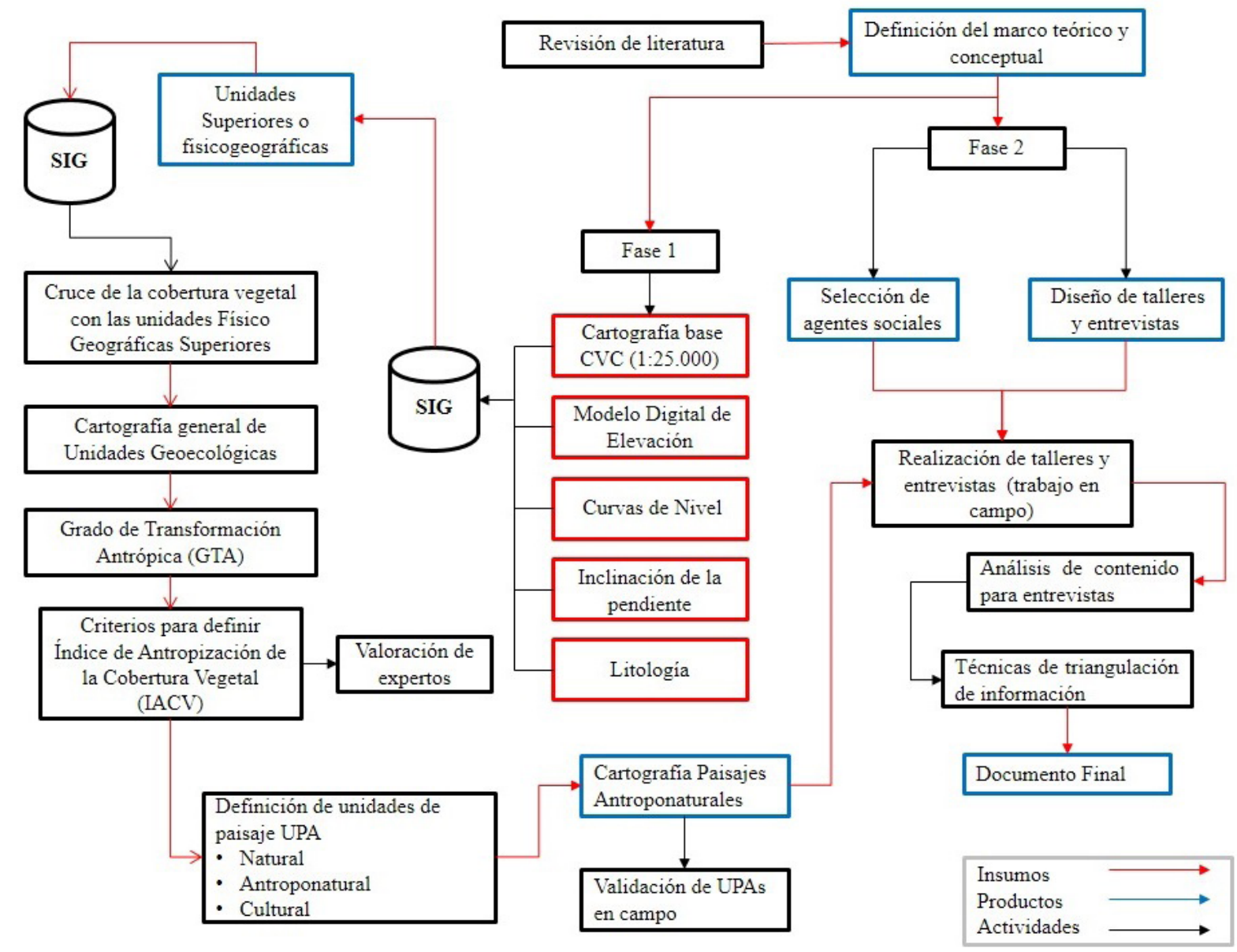

Figura 3. Metodología general para identificar la percepción.

Fuente: Elaboración propia. 
Marco Antonio Aguirre, Laura Isabel López Ibarra, Francy Viviana Bolaños Trochez,

Diego Fernando González Guevara, Oscar Buitrago Bermúdez

La entrevista empleada fue de tipo semiestructurada (Babbie, 1998; Flick, 2004; Létourneau, 2009; Martínez, 2006). Posteriormente, se diseñaron talleres de cartografía social donde los participantes identificaron aspectos asociados a los cambios en los paisajes y las condiciones ambientales del agua y los ecosistemas, tomando como punto de referencia temporal un margen de veinte años para que los participantes pudiesen rescatar vivencias y percepciones pasadas y presentes con respecto a cada una de las categorías analizadas en las entrevistas. Las entrevistas se interpretaron a partir del análisis de contenido. Al final, la información fue integrada y analizada empleando técnicas propias de las ciencias sociales, como los métodos de triangulación (Carvajal, 2006).

\section{Elementos de la percepción}

En esta parte del documento se presentan algunos de los resultados obtenidos hasta la fecha en el marco del proyecto. Para ello, se aborda la clasifi- cación de los paisajes antropogénicos en contraste con algunos de los elementos del análisis de contenido de las entrevistas y los talleres realizados en campo (Universidad del Valle, 2016c).

\subsection{Paisajes antropogénicos}

La Figura 4 evidencia que la mayor proporción de la cuenca hidrográfica se encuentra dentro de la tipología de paisajes antroponaturales (en color amarillo) localizados sobre toda la cuenca, en especial, en la parte baja que va desde Loboguerrero hasta la desembocadura, cerca al puerto de Buenaventura. En la Tabla 1 se presenta el porcentaje en hectáreas para cada una de las unidades de paisajes antroponaturales y es posible identificar un desequilibrio o desproporción en materia espacial, ya que las unidades asociadas a paisajes culturales y antroponaturales representan el 25,86\% y el $68,96 \%$ del área total de la cuenca. Los datos indican que la cuenca tiene un alto grado de intervención humana debido a las actividades económicas productivas (ver Figura 4).

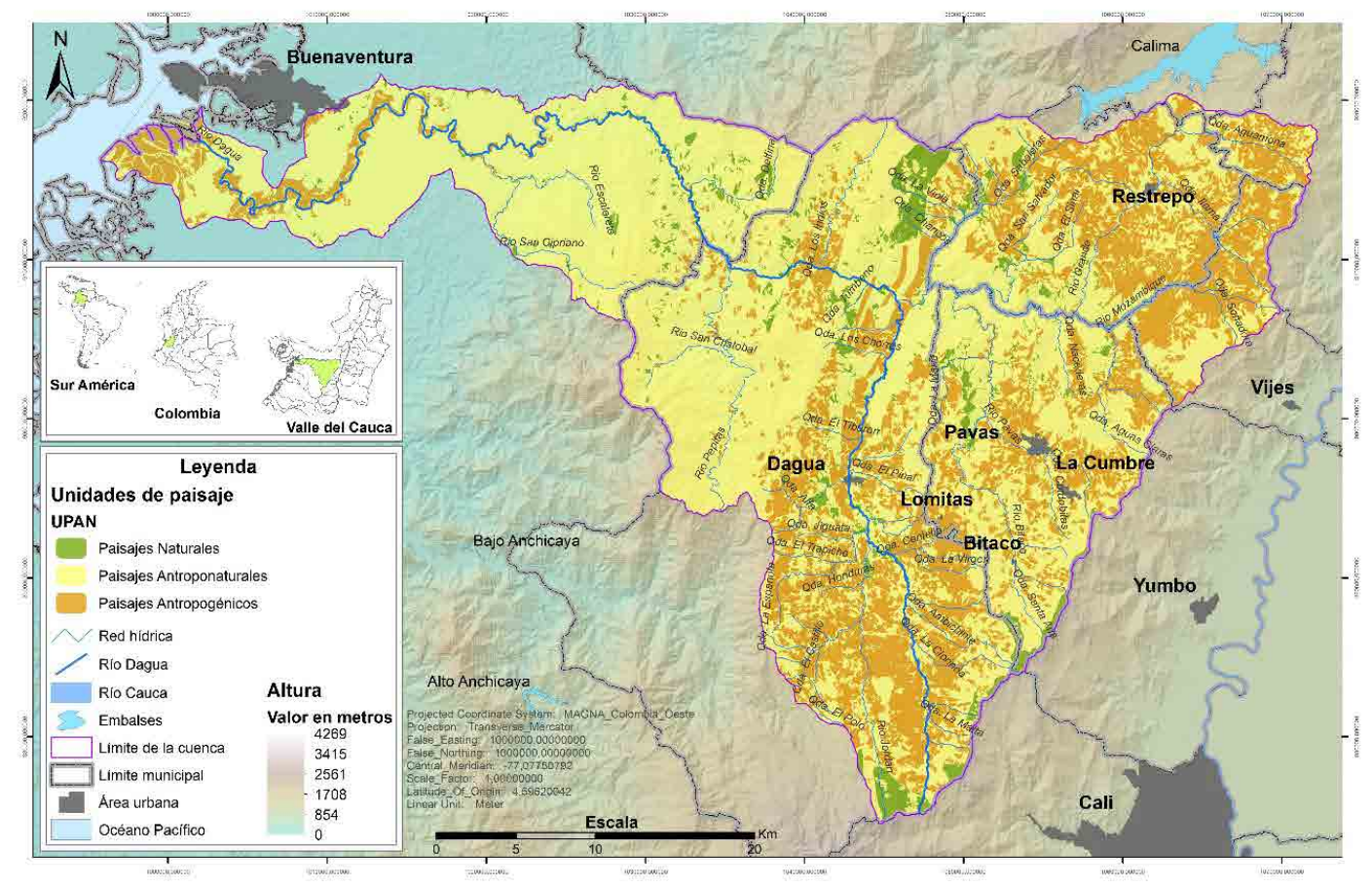

Figura 4. Paisajes antropogénicos de la cuenca del río Dagua.

Fuente: Elaboración propia. 
TABLA 1. RELACIÓN EN HECTÁREAS Y PORCENTAJES POR TIPOLOGÍA DE PAISAJE EN LA CUENCA

\begin{tabular}{|c|c|c|c|c|}
\hline Tipología de paisaje & $\begin{array}{l}\text { Total en } \\
\text { hectáreas }\end{array}$ & $\%$ en ha & $\begin{array}{l}\text { Grado de } \\
\text { intervención }\end{array}$ & $\begin{array}{l}\text { Coberturas del suelo destacadas en } \\
\text { cada unidad de paisaje }\end{array}$ \\
\hline $\begin{array}{l}\text { Paisajes culturales o } \\
\text { antropogénicos }\end{array}$ & $36.725,21$ & 25,86 & $\begin{array}{c}\text { Alto } \\
\text { (ámbar) }\end{array}$ & $\begin{array}{l}\text { Bosque fragmentado con pastos y } \\
\text { cultivos } \\
\text { Bosque manglar denso alto } \\
\text { Misceláneo de cultivos y espacios } \\
\text { naturales } \\
\text { Plantaciones forestales (pino y } \\
\text { eucalipto) } \\
\text { Centros poblados } \\
\text { Extracción de material de arrastre y } \\
\text { minería aurífera }\end{array}$ \\
\hline Paisajes antroponaturales & $97.926,35$ & 68,96 & Medio (amarillo) & $\begin{array}{l}\text { Bosque mixto fragmentado con pastos } \\
\text { y cultivos } \\
\text { Bosque fragmentado con vegetación } \\
\text { secundaria } \\
\text { Bosque alto y mixto de tierra firme } \\
\text { Pastos cultivados } \\
\text { Áreas naturales desnudas } \\
\text { Arbustales, pastos y rastrojos } \\
\text { Extracción de material de arrastre } \\
\text { Misceláneo de cultivos y espacios } \\
\text { naturales }\end{array}$ \\
\hline Paisajes naturales & $7.343,77$ & 5,17 & Bajo (verde) & $\begin{array}{l}\text { Bosque denso de tierra firme } \\
\text { Bosque mixto denso } \\
\text { Misceláneo de cultivos y espacios } \\
\text { naturales } \\
\text { Vegetación secundaria o en transición }\end{array}$ \\
\hline
\end{tabular}

Fuente: Elaboración propia.

En los paisajes antroponaturales (en color amari1lo) predominan coberturas del suelo asociadas a bosque fragmentado con vegetación secundaria y mosaicos de pastos y cultivos con espacios naturales. En la primera cobertura fue posible identificar usos del suelo como la extracción de materiales de arrastre para la ampliación de la vía Loboguerrero-Buenaventura y de metales preciosos de forma artesanal e ilegal. En la segunda cobertura se observó siembra de pastos para ganadería extensiva en ladera con presencia de cultivos limpios, como piña, pino, eucalipto y sábila. Los centros poblados de la cuenca hacen parte de este tipo de paisaje, el cual presenta grados significativos de intervención humana sobre el agua y los ecosistemas porque la mayoría de las viviendas depositan sus aguas residuales en las fuentes hídricas, ya que no disponen de sistemas sépticos ni de plantas de tratamiento de aguas doméstica (Universidad del Valle, 2016a). Es importante mencionar que en este tipo de paisaje es característica la intervención de zonas boscosas naturales para el desarrollo de las distintas actividades humanas, lo cual altera los ecosistemas presentes.

Los paisajes antropogénicos (en color ámbar) se localizan sobre la parte alta, en sentido norte-sur hasta la intersección de los ríos Dagua y Bitaco, a la altura de Loboguerrero. Esta tipología representa coberturas del suelo con altos grados de intervención antrópica. Al norte de la cuenca, en los municipios de Restrepo, Vijes y La Cumbre, estos paisajes concuerdan con áreas destinadas a cultivos de café, piña y plantaciones de pino y eucalipto en pendientes pronunciadas $(>25 \%)$, además de la presencia de empresas avícolas que han venido incrementando su concentración sobre 
Marco Antonio Aguirre, Laura Isabel López Ibarra, Francy Viviana Bolaños Trochez,

Diego Fernando González Guevara, Oscar Buitrago Bermúdez

esta parte de la cuenca. Hacia el sur, en la parte alta, sobre el municipio de Dagua, se destaca la presencia de actividades agropecuarias, entre las que resalta la localización de empresas avícolas y porcícolas, así como la siembra de hortalizas. Además, la presencia de viviendas campestres

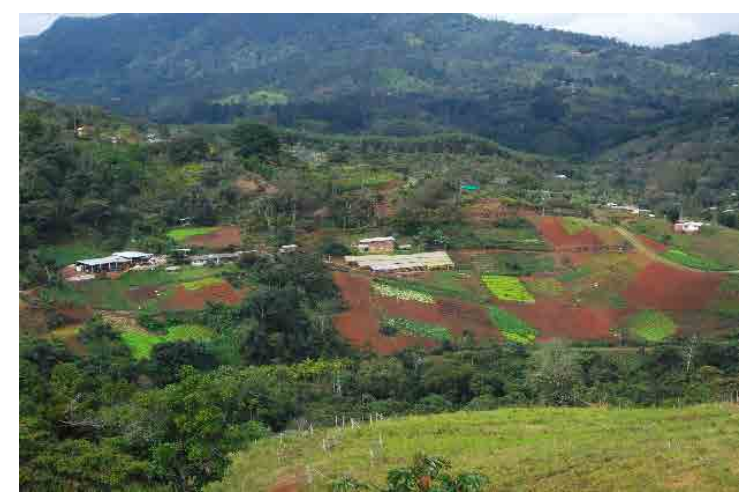

Figura 5. Visual de la parte alta de la cuenca: bosque fragmentado para expansión de cultivos.

Fuente: Equipo de trabajo, 2015.

En la parte baja se destacan algunas áreas asociadas a paisajes con altos grados de intervención humana ya que se presenta, según información secundaria y los agentes sociales entrevistados, extracción de madera y fragmentación del mangle de forma ilegal.

Los paisajes naturales (en color verde) comprenden la menor proporción espacial de la cuenca, con el 5,17\% del área total (ver Tabla 1), cifra que se traduce en 7.343 ha de las 144.000 que comprende la cuenca hidrográfica del río Dagua. Este tipo de paisajes corresponde a zonas de bosque, como la reserva forestal de Bitaco, localizada en la parte alta de la cuenca, al suroriente; fragmentos de bosque de la reserva de San Ciprino, en la parte baja, y áreas de conservación forestal localizadas al norte de Loboguerrero, cerca al límite municipal de Calima Darién. Las coberturas del suelo presentes en este paisaje se asocian a bosque denso de tierra firme, bosque fragmentado con pastos y cultivos, y vegetación secundaria o en transición. Los usos del suelo están ligados a prácticas agropecuarias con bajos grados de tecnificación y agricultura de subsistencia o pancoger. Actualmente, dichas dispersas y parcelaciones es un hecho espacial que evidencia la fragmentación de la tierra en la cuenca, debido a la presión ejercida por el área de metropolización de la ciudad de Santiago de Cali, al suroriente de la cuenca (ver Figuras 5 y $6)$.

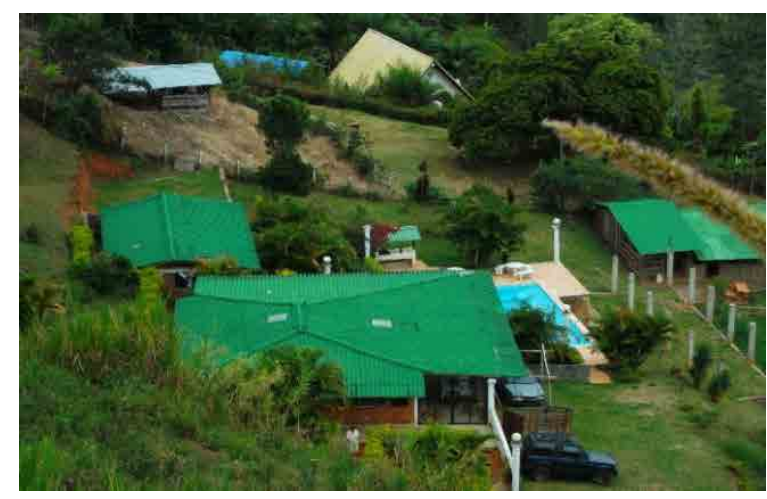

Figura 6. Visual de la parte alta de la cuenca:

construcción de fincas campestres en áreas de bosque denso alto y fragmentado.

Fuente: Equipo de trabajo, 2015.

áreas están siendo intervenidas con fines agropecuarios intensivos (presencia de monocultivos) y localización de viviendas campestres (ver Figuras 5 y 6); sin embargo, una de las mayores preocupaciones se relaciona con la presencia de actividades ilegales mineras y de extracción de madera.

\subsection{Percepción del paisaje}

Con respecto a las unidades de paisaje antropogénicas presentes en la cuenca, los agentes sociales entrevistados, a través de los talleres de cartografía social, manifestaron que el paisaje se encuentra altamente intervenido ya que, hasta la fecha, el número de viviendas presentes en el área se ha incrementado. Así mismo, evidenciaron un aumento progresivo en la presencia de actividades económicas agropecuarias, tales como la ganadería extensiva y la avicultura, además de grandes extensiones de tierra dedicadas a la extracción de materias primas para la industria del papel, asociadas a cultivos de pino y eucalipto. Esta postura concuerda con los talleres de cartografía social, donde los agentes participantes señalaron espa- 
cialmente dichas áreas como puntos de intervención humana significativos, ya que las prácticas presentes fomentaban el deterioro de ecosistemas

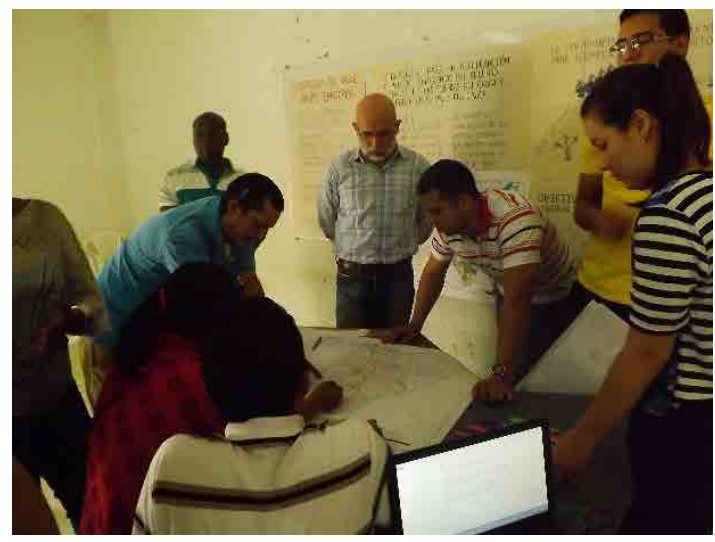

Figura 7. Talleres de cartografía social, parte alta de la cuenca.

Fuente: Equipo de trabajo, 2015.

En relación con la percepción del paisaje, el agua y los ecosistemas, uno de los agentes sociales entrevistados manifestó lo siguiente:

[...] en esta parte de la cuenca predominan las parcelaciones, los barrios nuevos [...], en los últimos años, el río ha perdido bastante caudal, está muy contaminado, los peces han disminuido, los puntos donde nace el agua han disminuido [...]. Hoy en día hay bosques primarios, pero están siendo talados. Las personas están sembrando cultivos limpios, lo cual acelera la erosión del suelo. Las cabeceras municipales están ampliando su frontera. La llegada de la empresa Cartón Colombia deterioró en un $90 \%$ los bosques y las especies de flora y fauna [...], hoy en día no se ve un guatín ni una guagua; anteriormente había tigrillo, tatabro, cusumbo; las culebras mapanás; hoy en día no existen, pues su hábitat se ha reducido (Entrevista con un agente social del municipio de Dagua, abril de 2016). y fuentes de agua. En las Figuras 7 y 8 se observan algunos de los talleres de cartografía social en la cuenca alta.



Figura 8. Talleres de cartografía social, parte baja de la cuenca.

Fuente: Equipo de trabajo, 2015.

De acuerdo con la percepción de los agentes sociales frente a los paisajes antroponaturales, se evidencian problemas de deforestación y sustitución de bosques naturales por plantaciones forestales promovidas principalmente, en la cuenca alta, por la empresa Cartón de Colombia. Del mismo modo, la constante adecuación de suelos para actividades agropecuarias como la ganadería intensiva en ladera y la construcción de parcelaciones campestres han generado el deterioro del vital líquido y los ecosistemas, debido a los procesos de contaminación asociados a los vertimientos de aguas domésticas, que se depositan directamente sobre ríos y quebradas sin ningún tipo de control por parte de las autoridades ambientales (Universidad del Valle, 2016a) (Figuras 9 y 10$)$. 


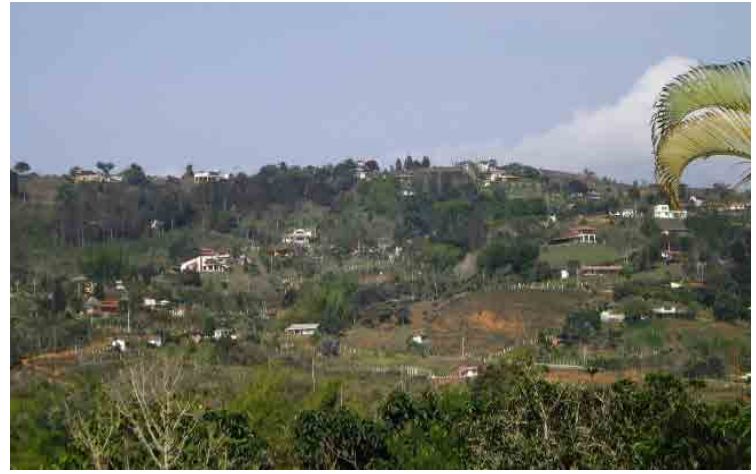

Figura 9. Fragmentación del suelo para construcción de fincas campestres, parte alta de la cuenca.

Fuente: Equipo de trabajo, 2015.

Con respecto a lo anterior, uno de los agentes sociales entrevistados menciona lo siguiente:

En la cuenca alta, los afluentes de agua se encuentran en mal estado, el tema de bosques también, pero por descuido de la autoridad ambiental CVC [Corporación Autónoma Regional del Valle del Cauca], pues dan autorizaciones a distintas personas sin mirar los daños que le están haciendo al ecosistema y al agua; entonces, se produce la deforestación y aparecen todas esas viviendas (Entrevista con un agente social en el municipio de Restrepo, abril de 2016).

[...] nosotros todavía tenemos bosque, todavía podemos decir que tenemos agua. En este momento se formó un comité hídrico para la defensa del agua, con el fin de optimizar el gasto del recurso hídrico, y digamos que dicha acción va a llevar a los múltiples actores en la cuenca a despertar y hacer mejor uso del vital líquido. Hay actividades económicas que impactan directamente el bosque y el agua, como la siembra de piña, pino y eucalipto, pues estas actividades requieren de tumbar bosque; otros dicen que las actividades que más impactan el paisaje y el agua son los cultivos forestales, pero todo impacta un poquito al recurso natural (Entrevista con un agente social en el municipio de Restrepo, abril de 2016)

Con respecto a la percepción de los paisajes naturales, los agentes sociales entrevistados manifiestan que esta coincide con las figuras de protección de bosques y zonas de abastecimiento de agua para acueductos de la cuenca, por lo cual promueven

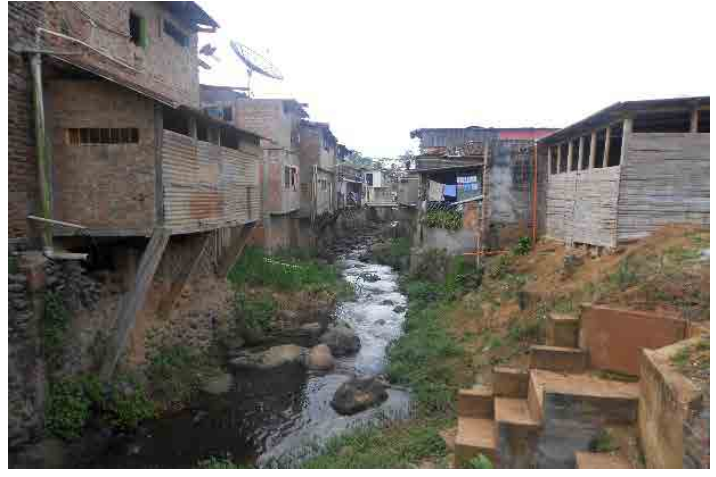

Figura 10. Vertimiento de aguas domésticas en las fuentes hídricas, parte alta de la cuenca.

Fuente: Equipo de trabajo, 2015.

estrategias de conservación en dichas áreas para garantizar la calidad y disponibilidad de agua. Adicionalmente, este tipo de paisajes, según los agentes sociales, se destacan por la no presencia de actividades humanas como la industria, la extracción de minerales y la agricultura, lo cual ha facilitado los procesos comunitarios de conservación. Sin embargo, a diferencia del resto de la cuenca, los agentes sociales señalan que en la parte alta la empresa Cartón de Colombia es la principal amenaza de las áreas de bosque y productoras de agua, ya que actualmente viene adelantando operaciones cerca de las áreas de protección forestal, principalmente en la zona correspondiente al municipio de Restrepo, donde se han generado impactos ambientales sobre el agua y los ecosistemas por el desarrollo de la actividad forestal (Universidad del Valle, 2016a). En palabras de algunos de los agentes sociales entrevistados, se evidencia lo siguiente:

Uno de los problemas más graves en la cuenca alta es que la empresa Cartón Colombia está comprando los suelos de toda la cordillera Occidental [...] la gente se acoge a cualquier propuesta y vende su tierrita [...] donde está Cartón Colombia el suelo se está secando, la fauna está disminuyendo, la flora ya no es diversa, el paisaje se va limitando a pinos y eucalipto; ese es un problema grave porque tiende agudizar el problema del agua. Además, está generando un impacto ambiental sobre la zona protectora y de amortiguación de la reserva. [...] Por otro lado, no se está produciendo comida, solo se están haciendo casas bonitas que consumen más 
agua, más energía, porque esa gente llega con sus aparatos, con sus piscinas y todo eso consume (Entrevista con agentes sociales en los municipios de Dagua y La Cumbre, abril de 2016).

La parte baja de la cuenca, según los agentes sociales entrevistados, presenta una situación de importancia, ya que la extracción minera de materiales de construcción o metales preciosos ha traído consigo problemáticas de carácter social y ambiental. Zaragoza, por ejemplo, es uno de los casos representativos en la cuenca y en el departamento (ver Figuras 11 y 12), ya que dicha actividad económica ha dejado secuelas irreparables



Figura 11. Minería aurífera ilegal en Zaragoza, sobre el cauce principal del río Dagua, cuenca baja.

Fuente: Sandoval (s. f., en Gobernación del Departamento del Valle del Cauca et al., 2014, p. 12).

Por otro lado, los actores institucionales entrevistados son conscientes de la importancia que tiene el agua para las comunidades de la cuenca baja del río Dagua, según lo manifiesta un funcionario de la Corporación Autónoma Regional del Valle del Cauca (CVC):

Para la gente de la zona y las comunidades el agua es todo, si usted va y les pregunta, yo sé que le van a decir lo mismo [...] la gente tiene su pensamiento y formas de vida arraigadas y en relación al agua, a raíz de lo anterior, nosotros como institución tenemos una dimensión de conservación frente a las comunidades y decimos que el agua siempre está ahí, de igual forma, que su relación con el agua es muy fuerte; si a esta gente le llegase a faltar el agua, yo creo que se mueren y más rápido que cualquier otra persona en la cuenca [...] actualmente estamos porque la extracción se realizó en casi $14 \mathrm{~km}$ sobre el cauce principal del río Dagua. Lo anterior se evidencia en una noticia del diario El País en el 2013: "Montículos de arena y piedra, cambuches abandonados y lagunas de aguas estancadas conforman la huella que dejó el devastador paso de más de 9.500 personas que durante tres años -2009 y 2012- trataron de sacar hasta la última pepita de oro" (El País, 2013). Actualmente, en la parte baja de la cuenca hidrográfica del río Dagua se lleva a cabo la extracción de materiales de arrastre del cauce principal para la ampliación de la doble calzada Loboguerrero-Buenaventura.

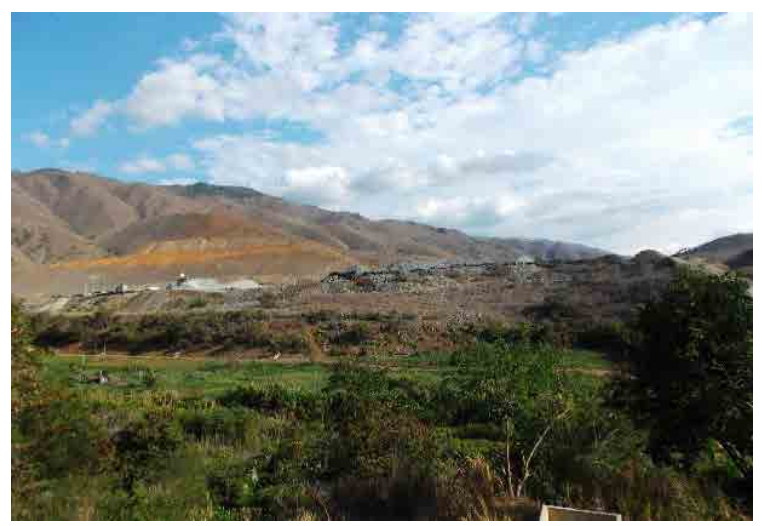

Figura 12. Extracción de materiales para construcción y ampliación de la doble calzada Buga-Buenaventura.

Fuente: Equipo de trabajo, 2015.

trabajando con ellos temas del agua, en relación a que es un recurso finito y que toca empezarlo a organizar y planificar (Entrevista con un agente social en el municipio de Dagua, abril de 2016).

En esta perspectiva, el agua y, en especial, los ríos constituyen el eje principal de las actividades de las comunidades étnicas presentes en la cuenca; son la base para su sustento, como el desarrollo de la pesca o de la minería artesanal, las actividades de recreo e incluso la materialización de labores domésticas (Universidad del Valle, 2016c). En la cuenca baja del río Dagua, los agentes sociales convocados a los talleres de cartografía social recordaron que hace más de veinte años la actividad agrícola tenía una mayor influencia en la zona y marcaba una gran diferencia en las formas de re- 
Marco Antonio Aguirre, Laura Isabel López Ibarra, Francy Viviana Bolaños Trochez,

Diego Fernando González Guevara, Oscar Buitrago Bermúdez

lación económica, pues estas se caracterizaban por lazos de solidaridad reflejados en el desarrollo de mingas campesinas en las cuales se intercambiaban productos entre los mismos habitantes (Universidad del Valle, 2016c).

En cuanto a las actividades económicas productivas, el corte de madera y la extracción minera eran las principales prácticas económicas en la cuenca baja: la primera se localizaba hacia la desembocadura del río Dagua, en el océano Pacífico, y contaba con la presencia de empresas como Cartón de Colombia y Concivil, dedicadas a la tala indiscriminada de bosques, específicamente en las veredas San Cipriano y Córdoba. La segunda se localizaba desde Cisneros hasta Zaragoza, sobre el cauce principal del Dagua, y los materiales obtenidos de estas actividades eran transportados por el río para su pronta comercialización.

En las entrevistas y talleres de cartografía social se reitera que la construcción de la vía Buga-Buenaventura ocasionó la pérdida de la navegabilidad del río Dagua, que hace más de cincuenta años permitía el transporte fluvial de mercancías desde la población de Juntas, en el municipio de Dagua, hasta la desembocadura del río. Sin embargo, con el ingreso del ferrocarril y la construcción de la vía, este medio de transporte fue reemplazado, pese a su utilidad para los nativos de la zona. Así lo expresa un funcionario de la Policía Nacional

Hace unos 50 años o más el río era navegable, desde la desembocadura hasta Juntas, localizado en el municipio de Dagua, la realización de este proyecto afectó ambiental y socialmente la cuenca del río; entonces, cuando ya venían levantando la vía fueron tirando todos esos materiales al río y fueron tapando el caudal, entonces de ahí para allá de ahí para acá fue sufriendo y padeciendo la población y sus comunidades porque las tierras y las vegas del río destinadas a la siembra de cultivos tradicionales se fueron perdiendo (Universidad del Valle, 2016c, p. 34).

Del mismo modo, los agentes sociales entrevistados mencionaron que hace veinte años se podían bañar y pescar en el río Dagua; sin embargo, desde el año 2000, debido a la alta contaminación producida por el depósito de residuos sólidos y el verti- miento de aguas domésticas, no se pudieron volver a realizar ese tipo de actividades. Las comunidades también destacan en los talleres el impacto ambiental y social que ha producido el basurero del Distrito de Buenaventura, localizado cerca a la quebrada El Venado, en la vereda de Córdoba. En las Figuras 13 y 14 se evidencian algunas de las situaciones ambientales presentes en la parte baja de la cuenca, identificadas previamente por comunidades locales a través de entrevistas y talleres de cartografía social.

En cuanto a la percepción del agua, las comunidades y los agentes entrevistados en la parte baja de la cuenca mencionan que la abundancia y la disponibilidad del vital líquido se ha convertido en un instrumento de doble vía, ya que, por un lado, evidencia la riqueza ambiental de la cuenca y, por otro, incide en la concepción que se tiene de esta, en especial porque las comunidades la representan como un recurso inagotable. Esta situación no ha facilitado la implementación de medidas orientadas a garantizar su uso racional y su conservación (Universidad del Valle, 2016c); en consecuencia, los esfuerzos emprendidos por las autoridades ambientales y municipales para el cuidado del agua no han sido eficientes debido a la falta de conciencia y de compromiso por parte de los pobladores. Además, esto ha conllevado a que las acciones de capacitación, concientización y trabajo en general para el cuidado del medio ambiente se hayan concentrado en la Reserva Forestal Protectora de los ríos San Cipriano y Escalerete, que se ha convertido en un ejemplo de recuperación mediante la concientización de comunidades, que han reemplazado sus actividades de explotación de los recursos naturales por el desarrollo de actividades ecoturísticas orientadas a fomentar la conservación y el cuidado de los servicios ecosistémicos. Así lo expresa la representante de la Fundación San Cipriano en una de las entrevistas realizadas en el corregimiento de Córdoba, en el municipio de Buenaventura:

El proceso se volvió digamos que auto-sostenible, tiende a volverse auto-sostenible en la medida que la reserva se convierte en fuente de generación de ingresos para las comunidades que están dentro de ella [...] aquí se vive 
a través del ecoturismo y a través del compromiso de la comunidad, si la comunidad no quiere no pasa nada, si la comunidad lo quiere, es rentable; antes era muy difícil, la gente estaba



Figura 13. Basurero, vereda Córdoba, municipio de Buenaventura: problemas con lixiviados en el cauce principal del río Dagua.

Fuente: Equipo de trabajo, 2015.

\section{Algunas conclusiones}

En general, se destaca la importancia de abordar problemáticas ambientales con perspectivas teóricas propias de la geografía, como la teoría de los paisajes antropogénicos, la cual ha permitido obtener miradas distintas a los métodos tradicionales de evaluación y planificación ambiental de las cuencas hidrográficas en Colombia. Metodológicamente, la propuesta logró incorporar técnicas de investigación cualitativas y cuantitativas en un mismo campo de acción investigativo. En términos prácticos, y según los intereses de la propuesta investigativa, el material compilado puede contribuir a las formas de organización social de base comunitaria para abordar procesos de gestión y planificación que integren las distintas percepciones existentes en torno al paisaje, el agua y los ecosistemas, de manera que se responda asertivamente a las realidades territoriales de la cuenca. Así mismo, se espera, en el mediano plazo, integrar los resultados de la investigación para formular y direccionar un plan de manejo del recurso hídrico y de conservación de suelos en la cuenca del río Dagua, según los lineamientos del proyecto de investigación bajo el cual se enmarca esta propuesta. desesperada no sabía de qué iba a sobrevivir y las únicas opciones eran a través de la minería y labores domésticas (Universidad del Valle, 2016c, p. 32).



Figura 14. Descarga de aguas domésticas y residuos sólidos al cauce principal del río Dagua.

Fuente: Equipo de trabajo, 2015.

En la cuenca hidrográfica del río Dagua los actores sociales perciben los paisajes antropogénicos de acuerdo al estado ambiental de la zona, los cambios en el paisaje y las acciones humanas que, al materializarse en prácticas espaciales, inciden de manera directa en la definición de cada tipo de paisaje en el tiempo. Además, a través de la percepción se identificaron diferentes problemáticas que actualmente afectan, en materia ambiental, el agua y los ecosistemas en la cuenca. Con respecto a lo anterior, en la parte baja se encontraron aspectos determinantes de la contaminación y del estado ambiental de la cuenca asociados a extracción de materiales de arrastre empleados en la construcción de la doble calzada Loboguerrero-Buenaventura y la extracción ilegal de minería de oro en el cauce principal del río Dagua. Dichas acciones humanas han propiciado dinámicas espaciales que han alterado las condiciones naturales de la cuenca en su parte baja, promoviendo, por ejemplo, la concentración de población sobre la vía principal, el aumento de residuos sólidos en los ríos y las quebradas y el vertimiento de metales pesados asociados a la explotación de oro, así como la alta intervención antrópica en los bosques de mangle para la extracción maderera y de fauna localizada en el área de transición entre aguas continentales y marinas. 
Marco Antonio Aguirre, Laura Isabel López Ibarra, Francy Viviana Bolaños Trochez,

Diego Fernando González Guevara, Oscar Buitrago Bermúdez

En la parte alta se identificó un aumento significativo de la fragmentación de la tierra debido a la presión ejercida por el área metropolitana de la ciudad de Santiago de Cali, ya que sus habitantes buscan zonas de recreo y descanso, con más frecuencia, los fines de semana. Por otro lado, las actividades agropecuarias como la ganadería extensiva en ladera y la sustitución paulatina de cultivos tradicionales como el café por cultivos limpios asociados a piña y sábila, y monocultivos de pino y eucalipto, han acelerado los procesos de erosión del suelo sobre la ladera e incidido directamente en la disponibilidad de agua para consumo humano, puesto que dichas actividades económicas demandan gran cantidad del vital líquido para su desarrollo. Lo anterior ha generado el deterioro de las fuentes hídricas y la pérdida de flora y fauna nativa de la cuenca. En esta perspectiva, los agentes institucionales, como la Corporación Autónoma Regional del Valle del Cauca (CVC) y las unidades municipales de asistencia técnica agropecuaria (Umata), coinciden en que dicho deterioro se debe principalmente al uso excesivo e inadecuado del suelo y el agua para el desarrollo de actividades humanas que deterioran los ecosistemas. De igual forma, se pone en evidencia la preocupación de las comunidades y los usuarios del agua frente al constante crecimiento de empresas avícolas en los municipios de Restrepo y Dagua ya que, por un lado, el desarrollo de tal práctica demanda de abundante acceso al agua, y, por otro, los residuos finales se depositan directamente en las fuentes hídricas.

Ante tal panorama, algunos de los agentes sociales manifiestan tener conocimiento y conciencia de los impactos generados social y ambientalmente como producto del desarrollo de las actividades mencionadas, así como de la débil función que desempeñan las autoridades ambientales del Estado en la cuenca, puesto que buena parte de las entrevistas indicaron un aumento en las problemáticas y conflictos socioambientales a raíz de algunas decisiones por parte de las autoridades ambientales, como la CVC y los entes delegados por los respectivos municipios. En palabras de uno de los agentes sociales entrevistados se evidencia lo siguiente:

La CVC dice que es la entidad que regula los recursos, que los administra, pero a veces le otorga concesiones de todo tipo a usted, a mí, sin ninguna justificación técnica. Además, el tema de bosques también es crítico en la cuenca, ya que la CVC otorgaba autorizaciones sin mirar los daños que se producirían en los ecosistemas, entonces, hay mucha deforestación. Por ejemplo, la CVC no hace cumplir las leyes ambientales, sobre todo con la empresa Cartón Colombia que deforesta mucho y no conserva las aguas. También, algunas entidades como las Umatas nos han colaborado muy poco con algunos recursos y asistencia técnica para mejorar las coberturas de bosque y conservación de agua, pero no hay una política bien centrada en la conservación de estos recursos en la cuenca (Entrevista con un agente social en el municipio de Dagua, agosto de 2016).

A la fecha, los planteamientos teóricos y la metodología propuesta han facilitado la identificación de los agentes sociales claves en la transformación del paisaje, el agua y los ecosistemas en la cuenca, así como la comprensión de sus acciones para producir dichos paisajes. Tal es el caso de la empresa Cartón de Colombia, cuya intervención en la parte alta ha modificado el paisaje y los ecosistemas debido a las extensas áreas que dispone para la siembra de pino y eucalipto. Del mismo modo lo han hecho las instituciones del Estado en su función de autoridad ambiental, puesto que se ven inmersas en gran parte de los debates asociados a problemas ambientales porque sus decisiones privilegian algunos sectores sociales en la cuenca, generando un detrimento de los procesos sociales desarrollados en torno al manejo y la conservación del agua y los ecosistemas.

Por otro lado, se destacan algunas formas de intervención y acción social comunitarias que buscan gestionar y conservar el agua a través de distintas figuras de organización, por ejemplo: grupos campesinos, mediante la figura de asociaciones de agua y usuarios que trabajan en pro y defensa de los recursos naturales; ONG ambientales, cuyos líderes pertenecen a las comunidades de la cuenca; instituciones educativas, mediante los programas de capacitación ambiental que se realizan en la cuenca, y los consejos comunitarios y resguardos indígenas que, a través de concepciones y prácticas culturales, han incidido sobre el agua y los ecosistemas, promoviendo otras formas de gestión y manejo de los recursos naturales. 


\section{Referencias}

Arias, P. (2003). Periferias y nueva ciudad. El problema del paisaje en los procesos de dispersión urbana. Sevilla, España: Secretariado de Publicaciones de la Universidad de Sevilla.

Babbie, E. (1998). Métodos de investigación por encuesta. Ciudad de México, México: Fondo de Cultura Económica.

Bolós, M. y Gómez, A. (2009). La ciencia del paisaje. Gestión del paisaje: manual de protección, gestión y ordenamiento del paisaje. Barcelona, España: Ariel.

Bolós, M. (1992). Manual de ciencia del paisaje. Teorías, métodos y aplicaciones. Barcelona, España: Masson.

Carvajal, A. (2006). Elementos de la investigación social aplicada. Cartagena de Indias, Colombia: Celes.

Corporación Autónoma Regional del Valle del Cauca (s. f.). Fichas ilustrativas de la situación ambiental de las cuencas hidrográficas del Valle del Cauca.

El País (28 de junio, 2013). Minería ilegal se traslada a la selva del pacífico. Recuperado de http://www.elpais.com.co/elpais/valle/noticias/mineria-ilegal-traslada-zaragozaselva-pacifico.

Espinoza, A. (2013). Paisajes antropo-naturales en Tzintzuntzan y sus alrededores (tesis de maestría). Universidad Nacional Autónoma de México, México. Recuperado de http://www.ciga.unam.mx/images/posgrado/tesis/espinozaa.pdf.

Flick, U. (2004). Introducción a la investigación cualitativa. Madrid, España: Morata.

Gobernación del Departamento del Valle del Cauca; Universidad del Valle; Pontificia Universidad Javeriana; Universidad Autónoma de Occidente; Acuavalle S.A. E.S.P.; Vallecaucana de Aguas S.A. E.S.P.; Corporación Autónoma Regional del Valle del Cauca (2014). Investigación recurso hídrico de las cuencas de los ríos Cauca y Dagua recuperado, Cali, Valle del Cauca, Occidente. Cali, Colombia.

Létourneau, J. (2009). La caja de herramientas del joven investigador: guía de iniciación al trabajo intelectual. Bogotá, Colombia: La Carreta Editores.

Lobato, R. (2014). Sobre agentes sociais, escala e produção do espaço: um texto para discussão. Em A. F. Alessandri Carlos, M. Lopes de Suouza e M. E. Beltrão Sposito (Org.). A produção do espaço urbano: agentes e processos, escalas e desafios (pp. 4151). São Paulo, Brasil: Contexto.

Martínez, M. (2006). Investigación cualitativa: síntesis conceptual. Revista de Investigación en Psicología, 9(1),123-146.

Martínez, C. (2012). El muestreo en investigación cualitativa: principios básicos y algunas controversias. Ciência \& Saúde Coletiva, 17(3), 613-619.

Mateo, J. M., Da Silva, E. V. y Cavalcanti, A. P. (2007). Geoecologia das paisagens. Uma visão geossistêmica da análise ambiental. Brasil: Editora Universidade Federal do Ceará (UFC). 
Marco Antonio Aguirre, Laura Isabel López Ibarra, Francy Viviana Bolaños Trochez,

Diego Fernando González Guevara, Oscar Buitrago Bermúdez

Mateo, J. M. (2008). Geografía de los paisajes. La Habana, Cuba: Universidad de La Habana.

Mateo, J. M., Da Silva, E. V. y Leal, A. (2012). Paisaje y geosistema: apuntes para una discusión teórica. Geonorte, 4(1), 249-260.

Mateo, J. M. (2014). La dimensión espacial del desarrollo sostenible: una visión desde América Latina. La Habana, Cuba: Universidad de La Habana.

Massochini, L. (2012). Gestão participativa no comitê da bacia hidrográfica do rio Araguari (MG). Em C. A. Di Mauro, V. Rosolen y V. de Oliveira Ferreira (Org.), Planejamento e gestão de recursos hídricos: exemplos mineiros. Uberlândia, Brasil: Assis Editora.

Priego, S. A. G., Bocco, G., Mendoza, M. y Garrido, A. (2008). Propuesta para la generación semiautomatizada de unidades de paisajes. Fundamentos y métodos. Ciudad de México, México: INE-SEMARNAT.

Santos, M. (2000). La naturaleza del espacio. Técnica y tiempo. Razón y emoción. Barcelona, España: Ariel.

Shishenko, P. G. (1988). Estabilidad de los paisajes a las cargas económicas. Geografía física aplicada. Kiev, Ucrania: Editorial de la Escuela Superior.

Universidad del Valle (2016a). Informe avance final marco teórico y metodológico actividad 1. Proyecto de Investigación Estrategias para la Recuperación y Manejo Integrado del Recurso Hídrico en las Cuencas del Cauca y Dagua, en el Valle del Cauca. En proceso editorial.

Universidad del Valle. (2016b) Informe diagnóstico de la gestión del agua en la cuenca del río Dagua. Proyecto de Investigación Estrategias para la Recuperación y Manejo Integrado del Recurso Hídrico en las Cuencas del Cauca y Dagua, en el Valle del Cauca. En proceso editorial.

Universidad del Valle. (2016c). Informe final análisis de contenido de entrevistas, talleres y aplicativo para identificar la percepción sobre el agua. Proyecto de Investigación Estrategias para la Recuperación y Manejo Integrado del Recurso Hídrico en las Cuencas del Cauca y Dagua, en el Valle del Cauca (p.34). En proceso editorial. 